\title{
Los derechos humanos en los textos clásicos y contemporáneos de las relaciones internacionales*
}

\section{Luis Ochoa Bilbao** \\ Rogelio Regalado Mujica***}

\section{RESUMEN}

El presente artículo ofrece los resultados del análisis crítico del discurso realizado a 42 libros clásicos y contemporáneos de las relaciones internacionales y la importancia de los derechos humanos en ellos. Este análisis del discurso académico demuestra que los derechos humanos no se han convertido en tema central de la teoría internacionalista. Por esta razón, se argumenta que no hay un cambio epistemológico ni ontológico en los textos internacionalistas para superar el enfoque Estadocéntrico de la disciplina.

PALABRAS CLAVE: Teoria de las relaciones internacionales, derechos humanos, análisis del discurso, Estadocentrismo.

\begin{abstract}
This article shows the results of the critical discourse analysis carried out to 42 classical and contemporary books on international relations and the importance of human rights. It is an analysis of the academic discourse that proves human rights are not central item of the internationalist theory. For this reason, it argues that there is neither epistemological nor ontological change in the internationalist texts that tries to overcome the State-centric approach of the discipline.
\end{abstract}

KEY WORD: international relations theory, human rights, discourse analysis, State-centric

* Artículo recibido el 14 de diciembre de 2016 y aceptado:27 de febrero de 2017.

** Internacionalista y sociólogo. Profesor de la licenciatura en Relaciones Internacionales en la Benemérita Universidad Autónoma de Puebla. Universidad Veracruzana, México. (anibalguz@yahoo.com.mx)

** Internacionalista egresado de la Benemérita Universidad Autónoma de Puebla (BUAP). Estudiante de la maestria en sociología del Instituto de Ciencias Sociales y Humanidades "Alfonso Vélez Pliego" de la BuAp. Profesor del Instituto de Ciencias Juridicas de Puebla, A. C. 


\section{SUMARIO}

1. Introducción

2. Derechos humanos: el dilema de la ruptura al paradigma científico de las relaciones internacionales y el discurso hegemónico anglosajón

3. El dilema del paradigma científico en las relaciones internacionales

4. Los clásicos de las relaciones internacionales y los derechos humanos

5. Los libros de texto para internacionalistas y los derechos humanos

6. Los libros sobre temas contemporáneos de relaciones internacionales y los derechos humanos

7. Reflexiones finales

\section{Introducción}

Para que los derechos humanos pasen de ser un ideal o una posibilidad y se cristalicen en la realidad, se deben cimbrar las estructuras culturales de las civilizaciones contemporáneas. Y esto será posible en la medida en que haya un "cambio de valores" en el seno de las sociedades. ${ }^{1}$ Se trata de un cambio de valores normativos y de aspiraciones universales, desde el lenguaje hasta los procesos judiciales y la convivencia diaria. Además, dicha transformación cultural debe ir siempre acompañada por las formulaciones teóricas y las evidencias empíricas que desde el ámbito académico se aporten.

Tomado en consideración el argumento anterior, el contenido del artículo parte de una pregunta central: ¿Existe un cambio de valores ontológicos (para explicar el mundo) y epistemológicos (acerca de cómo conocer mejor el mundo) en los textos de las relaciones internacionales que promueva a los derechos humanos como problema fundamental de la convivencia mundial? Entendemos aquí a los textos de las relaciones internacionales como los postulados de una disciplina científica de las ciencias sociales, conjunto que se ha desarrollado con cierto grado de intensidad desde la segunda mitad del siglo xx.

Si bien la disciplina de las relaciones internacionales tiene alcances globa202 les, la mayor parte de su corpus teórico proviene de la tradición anglosajona de las universidades británicas y estadounidenses. ${ }^{2}$ Esto nos permite señalar que hay una hegemonía discursiva y cultural en los textos de las relaciones internacionales. Si esto es así, si priva la perspectiva anglosajona en la disciplina,

\footnotetext{
1 Camacho, Daniel, "Relaciones internacionales, movimientos sociales y derechos humanos", en Mercedes de Vega (coord.), Diplomacia cultural, educación y derechos humanos, México, Secretaria de Relaciones Exteriores, 2011, p. 136. 2 Véase, Hoffmann, Stanley, "An American Social Science: International Relations", en Stanley Hoffmann (ed.), Janus and Minerva: Essays in the Theory and Practice of International Politics, Boulder, Vo., Westview Press, 1987.
} 
quizá también aparezca el tema de los derechos humanos ya que forma parte elemental del canon liberal democrático.

Sin embargo, el resultado de la investigación que ofrecemos en el artículo demuestra que no hay evidencia empírica para soportar el argumento de que los derechos humanos, ya sea como tema o como problema, formen parte central de los textos de las relaciones internacionales contemporáneas. Por el contrario, sigue siendo un problema marginal que no alcanza a competir en relevancia con los temas tradicionales de las relaciones internacionales, concretamente con la centralidad del Estado y sus atributos esenciales como el poder, la seguridad y la soberanía.

Este trabajo debe entenderse como un estudio del discurso académico de las relaciones internacionales. Los resultados presentados en este artículo provienen del análisis de 42 textos de relaciones internacionales escritos en inglés por autores estadounidenses, británicos y, en menor medida, australianos y canadienses. La selección fue hecha a partir de la ya mencionada hegemonía teórica del pensamiento anglosajón en el campo de las relaciones internacionales.

Los textos se agruparon en tres rubros: 1) los textos clásicos de relaciones internacionales publicados en el siglo xx; 2) los libros introductorios escritos para estudiantes universitarios que ofrecen una primera aproximación a las teorías y temas de las relaciones internacionales, publicados en los primeros años del siglo xxi y que se han convertido en "libros de texto" para politólogos e internacionalistas en gran parte de las universidades en occidente; 3) los libros sobre diversos temas de las relaciones internacionales que se han publicado en los primeros años del siglo xxI desde el ámbito académico anglosajón.

El diseño metodológico está basado en técnicas elementales del análisis crítico del discurso (ACD) que nos permiten ofrecer resultados cuantitativos y cualitativos. El análisis cuantitativo está determinado por el número de menciones a los derechos humanos que se hacen en cada texto. Esto nos ayuda a determinar si el concepto es, o no, central para los autores. De igual forma se investiga si hay capítulos enteros dedicados al tema de los derechos humanos en los textos revisados de política internacional.

$\mathrm{El}$ análisis cualitativo se aborda en tres frentes. El primero consiste en revisar si el tema de los derechos humanos es relevante o no en las obras de los autores clásicos y los más representativos de las teorías internacionalistas contemporáneas. El segundo revisa la relevancia que le otorgan los libros de texto a los derechos humanos. El tercero indaga sobre la amplitud y profundidad que los autores le dedican al análisis de los derechos humanos al presentar sus formulaciones teóricas sobre las relaciones internacionales contemporáneas. 
En los tres casos, se revisaron los vínculos entre las macroposiciones (los postulados fundamentales) y las proposiciones de coherencia global (los argumentos que sostienen los postulados fundamentales). Como se podrá apreciar, los derechos humanos prácticamente no aparecen como macroposiciones que replanteen un giro ni ontológico ni epistemológico en la disciplina de las relaciones internacionales.

Los derechos humanos son, más bien, proposiciones de coherencia global que sostienen una macroposición generalizada: que las relaciones internacionales contemporáneas son más complejas y variadas gracias a la emergencia de nuevos discursos de alcance humanitario como la protección del medio ambiente, el combate a la pobreza y los derechos humanos.

El artículo está conformado por cuatro apartados y unas conclusiones generales. En el primer apartado se presenta una reflexión sobre la hegemonía discursiva en las relaciones internacionales y lo que implicaría un cambio de paradigma. En el segundo apartado, se aportan las conclusiones sobre el ACD realizado a los libros clásicos de relaciones internacionales. En el tercer apartado, se ofrece el ACD realizado a los libros de texto publicados en el siglo XXI. En el cuarto apartado, se plantean los resultados obtenidos sobre los libros que abordan diversos temas de las relaciones internacionales en el siglo xxI.

\section{Derechos humanos: el dilema de la ruptura al paradigma científico de las relaciones internacionales y el discurso hegemónico anglosajón}

Hace ya más de seis décadas que el discurso de los derechos humanos ha sido difundido con un amplio esfuerzo. Es altamente comprensible que, para la segunda mitad del siglo xx, existiera una búsqueda implacable por evitar los horrores perpetrados durante las dos guerras mundiales. Ello abrió una veta sensible materializada en el entramado jurídico e institucional donde se identifican categorías éticas con pretensiones universales abanderadas por Occidente.

Precisamente los derechos humanos se gestan bajo esta lógica tal como los concebimos. Desde su aparición, fueron acompañados por una serie de críticas que cuestionan, entre otras cosas, la neutralidad de su confección. Asimismo, se les consideró un intento visible por homogeneizar el mundo en un orden evidentemente vertical y jerárquico que pretendía ser espejo de la cultura 0ccidental y justificación de la reproducción del capitalismo. ${ }^{3}$

\footnotetext{
${ }^{3}$ Las declaraciones sobre los derechos humanos por parte de la American Anthropological Association en 1947 y en 1999 avalan este argumento, por lo que se recomienda al lector su revisión detallada.
} 
No obstante, este discurso, que fue empleado como una herramienta para la contención del comunismo durante la Guerra Fría, contiene en sus entrañas un elemento subversivo y, actualmente, desafía tanto a sus creadores como a sus promotores más sagaces. Los derechos humanos no operan más como el fuerte de Occidente, sobre todo después de las inquietudes provocadas por el capitalismo rapaz. El innegable daño al medio ambiente provocado por el sistema de producción y consumo masivo, así como las crisis económicas, han evidenciado el rostro terriblemente imperfecto de los imbuidos por el triunfalismo liberal de la década de los noventa.

Es posible interpretar que, para los países occidentales, la visión de los derechos humanos adquiere discursivamente el sentido que Žižek, ${ }^{4}$ en diálogo con Lacan, expone con respecto a la ideología y sus aproximaciones al significante sin significado, el significante vacío. Esta apreciación se explica considerando que la identidad del significado se encuentra en la amplia gama de variaciones del significado mismo, lo cual discurre a lo largo de la enunciación elaborada, propiamente, por parte del sujeto. El papel que adquieren los derechos humanos en el discurso occidental se ha reformado como figura retórica.

En tiempos recientes, pareciera fácil situar a los derechos humanos como un punto de encuentro ya no solamente de las naciones centrales, sino también de los países periféricos y aspiracionistas que replantean su lugar en la historia. Sentado este contexto, y una vez aclarado el panorama desde el cual se pretende que se interpreten nuestros postulados, hemos de dar paso a la crítica del tema con relación a los paradigmas científicos.

\section{El dilema del paradigma científico en las relaciones internacionales}

A pesar de que a nivel político y mediático los derechos humanos han sido un tema recurrente en la agenda, en la academia la cuestión se esgrime de forma distinta; al menos así sucede en las relaciones internacionales. Esta disciplina surge de manera formal en la segunda mitad del siglo xx, momento en el cual repuntaba la necesidad de que un cuerpo de diplomáticos profesionales reconstruyeran el sistema mundial. De la misma forma, se debe de reconocer que la disciplina es una aportación principalmente anglosajona a las ciencias sociales, lo cual le da sentido a la metodología empleada en esta investigación. ${ }^{5}$

\footnotetext{
${ }^{4}$ Žižek, Slavoj, Ideología, un mapa de la cuestión, Buenos Aires, FCE, $2003 .$.

${ }^{5}$ Véase OchoA BILBAO, LuIs, La carrera de Relaciones Internacionales en México: origenes y situación actual, Ciudad de México, Colmex - BUAP, 2011.
} 
Al concentrarnos en el estudio de los contenidos temáticos en los libros de relaciones internacionales, pretendemos detectar en qué medida los derechos humanos han modificado la concepción del mundo de los textos académicos internacionalistas. Los libros elegidos para realizar el ACD tienen dos funciones primordiales: 1) ofrecer un marco interpretativo para explicar los fenómenos internacionales; y 2) proponer dicho marco interpretativo como herramienta teórica y metodológica para analizar y explicar cualquier fenómeno internacional.

El primer punto consiste en defender una perspectiva epistemológica en competencia con otras, es decir, proponer una herramienta para tratar de llegar a la verdad o, al menos, para realizar suposiciones validadas por una metodología objetiva y racional. ${ }^{6}$ El segundo punto consiste en diseminar tanto la metodología como la perspectiva en una función educadora, ya sea para explicar las relaciones internacionales o para justificar la eficacia de un marco teórico y de una metodología en particular.

Este esbozo nos introduce a una de las provocaciones más importantes para la ciencia en general: nos referimos a la ilustrada por Thomas Kuhn ${ }^{7}$ en su obra clásica La estructura de las revoluciones cientificas. El discurso de Kuhn indica que la historia de la ciencia ha sido planteada, tradicionalmente, de manera acumulativa. Sin embargo, existen iniciativas que se alejan diametralmente del componente historiográfico y presentan una perspectiva interpretativa desde la temporalidad en que se desarrollan las aportaciones científicas.

Así, Kuhn expone que, en determinados momentos, se plantean problemas que la ciencia "normal", con los métodos regulares, no puede resolver. Asimismo, hay situaciones en las cuales los resultados presentan anomalías y evidencian la pérdida de la brújula por parte de la ciencia misma. Cuando esto sucede, las investigaciones extraordinarias dan paso a lo que Kuhn denomina las revoluciones científicas: "Los episodios extraordinarios en que tienen lugar esos cambios de compromisos profesionales son los que se denominan en este ensayo revoluciones científicas. Son los complementos que rompen la tradición a la que está ligada la actividad de la ciencia normal". ${ }^{8}$

El momento de ruptura que plantea Kuhn, y las necesidades que lo hacen emerger, se están manifestando actualmente en las relaciones internacionales. Los problemas contemporáneos y las anomalías presentes en los resultados obtenidos a través de los métodos habitualmente empleados en la disciplina

\footnotetext{
${ }^{6}$ Véase Kornprobst, Markus, "International Relations as Rhetorical, Discipline: Toward (Re-)Newing Horizons", International Studies Review, núm. 11, 2009, p. 95.

${ }^{7}$ Kunn, Thomas, La estructura de las revoluciones científicas, México, FCE, 2004, p. 27.

${ }^{8}$ Kunn, Thomas, La estructura de las revoluciones cientificas, México, FCE, 2004, p. 27.
} 
exigen un cambio paradigmático que aún no se ha formulado. Asimismo, las perspectivas elaboradas para enfrentar las problemáticas relativas a la materia y contribuir a su solución surgieron a partir de exigencias determinadas por un contexto temporal específico.

Empero, este último se ha modificado rotundamente, provocando la necesidad de gestar nuevos entendimientos que operen con mayor efectividad. Esta provocación inicial sobre el posible cambio paradigmático plantea que, en la labor intelectual, los derechos humanos se puedan considerar temas de relevancia central de manera incisiva y no abordados periféricamente como ocurre en la actualidad. Tal es el caso en los textos clásicos y contemporáneos de relaciones internacionales.

Como se verá, los derechos humanos ni han sido paradigmáticos -en el sentido más laxo del concepto kuhniano-, ni se han convertido en macroposiciones epistemológicas dentro de los textos internacionalistas. En estricto sentido, no se ha abandonado la visión Estadocéntrica en las relaciones internacionales. Ésta tampoco ha sido sustituida por una visión humano-céntrica, como clama la perspectiva liberal que sostiene el entramado epistemológico de los derechos humanos.

El discurso académico sobre las relaciones internacionales se ha nutrido de diversas fuentes y espacios. No obstante, el mundo anglosajón tiene un lugar privilegiado en este entramado. La afirmación cobra sentido cuando se realiza un vínculo con el marco en el que fue desarrollado el conjunto teórico inicial de la disciplina y las voluntades políticas que orientaban su configuración. Este déficit se ha señalado también desde la académica de la periferia al mundo occidental. ${ }^{9}$

Es importante resaltar el carácter hegemónico anglosajón de los textos de relaciones internacionales porque, se supone, la promoción y difusión de los derechos humanos ha sido un estandarte ideológico de Occidente desde la segunda mitad del siglo xx. De ser así, los textos internacionalistas revisados en este trabajo habrían hecho de los derechos humanos un tema central de sus abordajes teóricos y descriptivos.

A partir de esta perspectiva, se analiza el papel de la perspectiva anglosajona dominante de relaciones internacionales y su relación con el concepto de hegemonía planteado por Gramsci. Mirta Giacaglia ${ }^{10}$ indica que la hegemonía de Gramsci es definida como “dirección política, intelectual y moral”. A esto le

\footnotetext{
${ }^{9}$ Véase Acharya, Amitav y Buzan, Barry, Non-Western International Relations Theory. Perspectives on and beyond Asia, New York - London, Routledge, 2010.

${ }^{10}$ Giacaglia, Mirta, "Hegemonia. Concepto clave para pensar la política", Tópicos, núm. 10, 2002, pp.151-159.
} 
agrega una división entre la política, por un lado, y la intelectual y moral por el otro. La primera, la política, consiste en "la capacidad que tiene una clase dominante de articular con sus intereses los de otros grupos”, lo cual la convierte en el elemento rector de la voluntad colectiva. En segundo lugar, en lo concerniente a la dirección intelectual y moral, explica que las consideraciones ideológicas deben ser tomadas en cuenta para posibilitar la constitución de dicha voluntad colectiva.

Precisamente, es la ideología, según Giacaglia, lo que cobra un rol decisivo y distanciado de la concepción tradicional marxista en la teoría de Gramsci. Esta concepción de la ideología “constituye un todo orgánico y relacional encarnado en aparatos e instituciones, un cemento orgánico que unifica en torno a ciertos principios articulatorios básicos un 'bloque histórico' y las prácticas productoras de subjetividades en el proceso de transformación social”. ${ }^{11}$ De esta manera, para lograr la transformación social, se parte de la percepción del sujeto en determinada concepción del mundo que también debe ser transformada.

Por lo tanto, los textos de relaciones internacionales difunden una concepción del mundo que se sitúa en un papel hegemónico. En el plano de la ideología, se articula un bloque histórico y se construyen subjetividades. Éstas, a su vez, responden a una dirección política que funge como elemento rector de la voluntad colectiva. En este plano, se ha reproducido una visión, cuyos conceptos y categorías constriñen las interpretaciones a fluctuaciones de relaciones de poder Estadocéntricas. Estas relaciones no promueven una visión incluyente de asuntos que se escapan de esta lógica y que resultan subversivos para la reproducción social del sistema tal y como se articula actualmente. En los siguientes bloques se intenta evidenciar esta crítica en los textos de relaciones internacionales, con base en los derechos humanos.

\section{Los clásicos de las relaciones internacionales y los derechos humanos}

Los textos que denominamos clásicos de las de las relaciones internacionales son fundamentalmente teóricos y fueron publicados durante la segunda mitad del siglo xx. Para la elaboración de este apartado se revisaron, bajo la metodología del análisis crítico del discurso, diez libros tradicionales de la disciplina. Estos libros aparecieron en el mundo anglosajón, y muy notoriamente en Estados Unidos. (véase cuadro 1).

\footnotetext{
"Giacagla, Mirta, "Hegemonía. Concepto clave para pensar la política", Tópicos, núm. 10, 2002, p.154.
} 
Cuadro 1

Autores clásicos de las relaciones internacionales

1 Hans Morgenthau, Politics Among Nations, 1948

2 Kenneth Waltz, Men, State and War. A theoretical Analysis, 1959

3 Robert Keohane \&t Joseph Nye Jr., Power and Interdependence, 1977

4 Kenneth Waltz, Theory of International Politics, 1979

5 Robert Gilping, War and Change in World Politics, 1981

6 Paul Kennedy, The Rise and Fall of the Great Powers, 1988

7 Cynthia Enloe, Bananas, Beaches and Bases: Making Feminist Sense of International Politics, 1989

8 Francis Fukuyama, The End of History and the Last Man, 1992

9 Samuel P. Huntington, The Clash of Civilizations and the Remaking of World Order, 1996

10 Alexander Wendt, Social Theory of International Politics, 1999

Las dos guerras mundiales y el reordenamiento del poder mundial motivaron que los temas internacionales incrementaran su interés en las universidades. La política internacional se estudiaba como parte de la ciencia política o de las ciencias de gobierno. Por lo tanto, los estudios internacionales deben considerarse como una rama de la ciencia política, afirmación que sigue siendo motivo de debate en la actualidad. ¿Son las relaciones internacionales una disciplina autónoma de la ciencia política o una disciplina subsidiaria de la misma?

En estricto sentido, nosotros aceptamos la visión de que el estudio de la política internacional o de las relaciones internacionales es subsidiario de la ciencia política. Y las razones son obvias: 1) los estudios internacionales en la enseñanza universitaria en Gran Bretaña y Estados Unidos a mediados del siglo xx no eran independientes de los departamentos de ciencias políticas; 2) los temas clásicos de las relaciones internacionales fueron los temas clásicos de la ciencia política, fundamentalmente el poder, la soberanía y la guerra; 3) las teorías clásicas de las relaciones internacionales son reformulaciones de las teorías clásicas de la ciencia política como el realismo, el liberalismo y el marxismo; 4) las metodologías de investigación son fundamentalmente politológicas; 5) algunos autores clásicos de las relaciones internacionales fueron politólogos como Hans Morgenthau, educado en Alemania, Kenneth Waltz, economista de origen y politólogo graduado de la Universidad de Columbia, Stanley Hoffmann educado en París en la École des Hautes Études en Sciences Sociales, y Karl W. Deutsch, formado en Praga. 
Finalmente, todas las elucubraciones de la ciencia política terminan siendo adaptadas por los internacionalistas a su campo de estudio. Sin embargo, no hay evidencia de una aportación contundente de las relaciones internacionales que se haya vuelto central para la ciencia política. Y lo mismo se puede decir de las demás ciencias sociales. En su carácter multidisciplinario, las relaciones internacionales se han nutrido de las metodologías y perspectivas teóricas de la historia, la economía, los estudios jurídicos, la sociología y la filosofía. Por ejemplo, los internacionalistas han adoptado en sus textos las aportaciones epistemológicas de Antonio Gramsci, Raymond Aron, Michel Foucault, Jürgen Habermas y Jacques Derrida. Pero no ha ocurrido a la inversa: ni los filósofos ni los científicos sociales citan como fundamentales a los autores identificados como internacionalistas.

Lo anterior nos permite emitir un primer argumento: las teorías de las relaciones internacionales son subsidiarias de los grandes temas teóricos y metodológicos de las ciencias sociales. Particularmente, en los autores clásicos de las relaciones internacionales, fueron preponderantes los intereses teóricos por explicar la estructura del sistema internacional, tema emanado del marxismo y de la sociología funcional estructuralista. Asimismo, se buscó explicar el poder y la guerra -citando siempre a Tucídides, Maquiavelo y Hobbes-; la riqueza -con Adam Smith como precursor inevitable- y el liberalismo institucional -basado siempre en Locke y Kant-.

En esta síntesis temática de las relaciones internacionales, es importante reiterar que, así como se aprecia una hegemonía anglosajona, también se aprecia una hegemonía del realismo como perspectiva explicativa. Tanto es así que todas las demás formulaciones teóricas en la disciplina son contestatarias del realismo. Éste es el caso del liberalismo institucional, el constructivismo, la teoría crítica, el posmodernismo, el feminismo y el cosmopolitanismo.

Respecto al interés por los derechos humanos, ocurre en las relaciones internacionales lo mismo que en las demás ciencias sociales: irrumpen como una novedad, como un tema distorsionador de la centralidad del poder. Los dere210 chos humanos resultan un elemento tan amplio como difuso, que sugiere la emergencia de un poder ciudadano o civil que se pretende global.

Los derechos humanos son, en esencia, la búsqueda por limitar jurídica y efectivamente el poder de la autoridad estatal en su capacidad disruptiva de la norma aceptada y la promoción de la convivencia no violenta. Pero, en las teorías clásicas de las relaciones internacionales, el Estado fue siempre el actor central de la guerra y la paz y la preocupación central era describir, explicar, diagnosticar y prescribir los modos y los medios al alcance del Estado para 
mantener su vigencia, su soberanía y su eficacia.

Por lo tanto, los derechos humanos fueron, irremediablemente, temas marginales para los autores clásicos de las relaciones internacionales, quienes siempre se vieron seducidos por explicar el poder, la fuerza, la soberanía y la seguridad. Sin embargo, incluso para el autor fundacional del realismo internacionalista, Hans Morgenthau, la ética en el quehacer político y la dignidad humanas no sólo no pasaron desapercibidas, sino que fueron elementales para su descripción del sistema internacional.

Morgenthau publicó su libro Politics Among Nations en 1948, en los prolegómenos de la Segunda Guerra Mundial. Las primeras partes del libro están dedicadas a la lucha por el poder en la política internacional, así como a la esencia y los elementos del poder estatal. Pero, en la quinta parte, aborda las limitaciones del poder internacional y, para explicarlo, trabaja los temas de la "moralidad internacional" y la opinión pública. Ambos están siempre supeditados al poder.

La ética y la moralidad en el pensamiento occidental, dice Morgenthau, corresponden siempre a los límites y las fronteras socialmente aceptables que la búsqueda del poder debe tener. ${ }^{12} \mathrm{Y}$, para las civilizaciones occidentales, el gran logro ha sido que la lucha por el poder político a nivel doméstico se haya reglamentado y pacificado. ${ }^{13}$

¿En qué medida se pueden replicar tales éxitos en la lucha por el poder en el sistema internacional? La moralidad en el sistema internacional -ya sea la protección de la vida humana en tiempos de paz o de guerra, o condenar la guerra como injusta o evitar la guerra total y los crímenes masivos- siempre estará sometida a los intereses nacionales, bajo el propósito de alcanzar y maximizar los intereses de Estados en su lucha por la supremacía. Si el mayor interés de una nación poderosa fuera eliminar la guerra, entonces su actuar moral justificaría la paz. Esto es, la moral respondería a un momento específico en la historia y a las condiciones sociales que la justificarían.

Para Morgenthau, los principios morales son aplicables a la política internacional porque todas las acciones humanas están sometidas a juicios morales. ${ }^{14}$ Sin embargo, considera que es diferente tratar de universalizar los criterios morales de un pueblo o de una civilización en particular, como los criterios morales que todos los pueblos debieran seguir, porque hay una gran brecha

\footnotetext{
${ }^{12}$ Morgenthau, Hans, Politics Among Nations, New York, Alfred A. Knopf, 1948, p. 169.

${ }^{13}$ Morgenthau, Hans, Politics Among Nations, New York, Alfred A. Knopf, 1948, p. 173.

14 Morgenthau, Hans, Human Rights and Foreign Policy, New York, Council on Religion and International Affairs, 1979, p. 1.
} 
entre la forma de juzgar los actos que uno mismo hace y la forma de juzgar los actos de los demás. ${ }^{15}$

Morgentahu propone una explicación relativista de los derechos humanos, por considerarlos una formulación abstracta, producto de una sociedad en particular, en un tiempo histórico determinado. Por lo tanto, los derechos humanos son variables y mutables. Morgenthau no los recomienda como vara sancionadora de los actos de otros pueblos y sociedades. ${ }^{16}$ Estos argumentos deben entenderse como la oposición que tomó Morgenthau al hecho de que los derechos humanos se convirtieran en una herramienta de política exterior.

Para Morgenthau, el ideal wilsoniano de la paz y la universalidad de los derechos humanos era incompatible con la verdadera aspiración de toda nación: la búsqueda de poder y la satisfacción de sus intereses. La conclusión de Morgenthau era que los derechos humanos no podían universalizarse por ser un concepto abstracto y relativista y porque, cuando los derechos humanos entran en conflicto con otros intereses, de manera pragmática, los Estados dejarían de lado los derechos humanos para perseguir intereses mayores a éstos.

Los planteamientos de Morgenthau parecerían, a primera vista, contrarios al ideal universalista de los derechos humanos. Pero, en el contexto histórico de sus ideas expresadas en "Human Rigths and Foreign Policy" en 1979, el equilibrio de poder estaba dividido entre dos potencias nucleares antagonistas. La lucha por la supremacía enmarcaba la política internacional. Del argumento relativista de Morgentahu, en una lectura optimista, se podría derivar que, en cualquier momento, la protección de los derechos humanos podría convertirse en la bandera política e ideológica de una nación poderosa o de un conglomerado de naciones poderosas.

Al transformarse en el "gran interés nacional o civilizatorio" de uno o varios Estados, los derechos humanos quizá alcanzarían la centralidad de la política internacional. Pero ese planteamiento sería más bien pos-Morgenthau, en un mundo globalizado y con leyes transnacionales que convirtieran a los Estados en colaboradores y no competidores. Esto es algo que ni los autores 212 clásicos ni los contemporáneos de las relaciones internacionales avizoran en un futuro cercano.

Otro texto clásico del realismo es el publicado en 1981, por Robert Gilpin: War and Change in World Politics. El tema central del texto es el cambio en

\footnotetext{
${ }^{15}$ Morgenthau, Hans, Human Rights and Foreign Policy, New York, Council on Religion and International Affairs, 1979, p. 3.

${ }^{16}$ Morgenthau, Hans, Human Rights and Foreign Policy, New York, Council on Religion and International Affairs, 1979, pp. 4-5.
} 
el sistema internacional que permite el surgimiento o el declive de los Estados nacionales. Su preocupación central es el poder y su manejo adecuado para mantener la preeminencia de un Estado nacional sobre los demás. El tema del mercado aparece como algo viable gracias a la presencia de un Estado que lo posibilite o lo limite. ${ }^{17}$

Para Gilpin, la interacción humana requiere de reglas que se consagran en derechos y cuya función es, sencillamente, solucionar los conflictos que surgen ante la distribución imperfecta de bienes. ${ }^{18}$ En su libro no aparece una sola mención a los derechos humanos. Ni como macroposición ni como proposición de coherencia global. Sencillamente, no es un tema que requiera atención. Para Giplin, la estabilidad del sistema internacional no respondía, en 1980, a una transformación paradigmática de la política internacional, sino a la relativa estabilidad del régimen de la bipolaridad. En él, lo predominante era la manera en que Estados Unidos y la Unión Soviética habían logrado equilibrar el poder hegemónico compartido.

Lo mismo ocurre con otro texto clásico de historia de las relaciones internacionales, publicado en 1988 por Paul Kennedy: The Rise and Fall of the Greate Powers. El autor se concentra en una visión materialista de la política internacional, con base en el ascenso y el declive de las potencias mundiales mediante el poder militar y el cambio económico. Los derechos humanos se mencionan como contenido de los Acuerdos de Helsinki en 1975 y como derechos violados por los regímenes autoritarios de derecha, apoyados por Washington durante la década de los setenta. Estas menciones sólo servían para ilustrar el fracaso del idealismo de la política de Jimmy Carter. ${ }^{19}$

El contrapeso al realismo ha estado del lado del idealismo y de las teorías internacionalistas más funcionalistas, como el liberalismo o el liberalismo institucional. La idea general de esta perspectiva es que hay una especie de "sociedad internacional" que, si bien no se encuentra sometida al régimen de la ley de una autoridad absoluta, sí se expresa en una serie de relaciones cooperativas entre los Estados nacionales. ${ }^{20}$

El texto emblemático de esta perspectiva es autoría de Robert 0. Keohane y Joseph S. Nye Jr., Power and Interdependence, publicado en 1977. El eje de

\footnotetext{
${ }^{17}$ Griffiths, Martin, Roach, Steven y Solomon, Scom, Fifty Key Thinkers in International Relations, New York - London, Routledge, 1999, pp. 16-17.

${ }^{18}$ Gilpin, Robert, War and Change in World Politics, Cambridge, Cambridge University Press, 1981, p. 34.

${ }^{19}$ Kenedy, Paul, The Rise and Fall of the Great Powers, New York, Random House, 1987, pp. 402, 401.

${ }^{20}$ Weber, CYNTHia, International Relations Theory: A critical Introduction, London \& New York, Routledge, 2005.
} 
su argumentación está en contra del realismo. Para estos autores, el concepto de "interdependencia compleja" contribuye a explicar la insuficiencia del paradigma realista en el análisis detallado de la toma de decisiones de los actores políticos.

El análisis de contenido del libro, en su cuarta edición publicado en 2012, arrojó que el concepto de derechos humanos sólo se menciona tres veces. Totalmente marginal, el concepto sirvió para ilustrar lo que los autores señalarían como la sensibilización de la opinión pública ante acciones criminales del Estado perpetradas en Somalia, Sudán del Sur o Kosovo.

Para Keohane y Nye, el discurso de los derechos humanos irrumpía en la opinión pública en la era de la información y tenía como objetivo presionar a los actores políticos (occidentales) a tomar medidas en casos de crisis humanitarias. ${ }^{21}$ Y Sólo eso. Para Keohane y Nye, los derechos humanos ya fueran como movimiento civil global, como norma consagrada en el entramado jurídico internacional o como aspiración para la convivencia, no servían para explicar las transformaciones contemporáneas del poder y la interdependencia en la política internacional.

La misma marginalidad se encuentra en otro libro fundamental de las teorías internacionalistas, Social Theory and International Politics, escrito por Alexander Wendt y publicado en 1999. Su importancia se debe a que Wendt ofrece una interpretación constructivista de las relaciones internacionales y trata, por ende, de resaltar la importancia de la subjetividad en la toma de decisiones de los actores políticos internacionales. Como el resto de las teorías contemporáneas de las relaciones internacionales, el constructivismo de Wendt trata de erosionar el rol hegemónico del realismo.

Lo hace mediante dos vías: 1) la crítica a la noción unitaria del Estado, como si éste fuera un actor fácil de asir y no estuviera exento de contradicciones o conflictos entre quienes personifican al Estado; 2) la crítica a la noción de que el concepto de anarquía no es una realidad dada, como plantean los realistas, sino una idea concebida por los tomadores de decisiones. Por lo tanto, a partir de Wendt, se pueden cuestionar seriamente las nociones de interés nacional, como si éste fuera uno solo para cada Estado nacional, y de seguridad, como si éste también fuera uno solo para los tomadores de decisiones de un Estado o para la población de una nación.

En el ACD, desde el punto de vista cuantitativo, Wendt sólo menciona dos veces a los derechos humanos. Desde el punto de vista cualitativo, sólo los usa

\footnotetext{
${ }^{21}$ Keohane, Rober \& Nye, Joseph, Power and Interdependence, New York, Longman, 2012, pp. 220-229.
} 
como ejemplo, junto con el comercio, de la agenda progresista de actores no estatales que pudieran modificar la centralidad del Estado en la política internacional. Wendt menciona en las conclusiones de su libro que su objetivo era hacer una ontología de las relaciones internacionales. Esto implica formular supuestos metafísicos que permitan explicar cómo funciona el sistema internacional, cómo está compuesto y cómo está estructurado. ${ }^{22}$

La ontología dominante en el mainstream internacionalista, dice Wendt, es materialista. Él propone una aproximación fenomenológica para defender una teoría idealista de las relaciones internacionales. ${ }^{23}$ La ontología de las relaciones internacionales de Wendt es "social", en el sentido de que "es mediante las ideas que los Estados se relacionan entre ellos y 'construccionista' en el sentido de que esas ideas ayudan a definir quiénes y qué son los Estados". ${ }^{24}$

Se puede señalar que el libro de Wendt ha sido realmente influyente en las teorías de las relaciones internacionales, al grado de que ha provocado un interés por las formas que adopta la política internacional desde la relevancia de la subjetividad de los actores. Por ello, hemos pasado a estudios internacionalistas menos descriptivos.

En este sentido, la subjetividad y el relativismo moral y cultural aparecen en la escena teórica internacionalista para discutir los alcances y límites de fenómenos tales como la migración, el racismo, el etno-nacionalismo, la globalización de la cultura del entretenimiento, del mercado y del ocio y la emergencia de una sociedad civil internacional progresista en la convivencia multicultural y cosmopolita. Todo este marco científico, quizá apuntalado por Wendt, tocará el tema de los derechos humanos. Pero, al igual que Wendt, hasta el momento, no ha propiciado una revolución ni ontológica ni epistemológica.

Otro autor, cuyos textos son considerados clásicos, es Kenneth Waltz. Con sus dos prolíficos libros, Man, State and War. A theoretical analysis ${ }^{25} \mathrm{y}$ Theory of International Politics, ${ }^{26}$ se posicionó como uno de los autores más relevantes para la disciplina. Contribuyó al campo del neorrealismo y se discutió altamente por sus perspectivas sobre las armas nucleares. Las críticas de Waltz, a la visión realista sobre la naturaleza humana, dotaron de un enfoque mucho más profundo, respaldado en el estructuralismo estadounidense que también estaba en boga por aquellos años.

\footnotetext{
22 Wendt, AleXander, Social Theory of International Politics, Cambridge, Cambridge University Press, 1999, p. 370.

${ }^{23}$ Wendt, AleXander, Social Theory of International Politics, Cambridge, Cambridge University Press, 1999, p. 371.

${ }^{24}$ Wendt, AleXander, Social Theory of International Politics, Cambridge, Cambridge University Press, 1999, p. 372

${ }^{25}$ Waltz, Kenneth, Man, state and war. A theoretical analysis, New York, Columbia University Press, 1959.

${ }^{26}$ Waltz, Kenneth, Theory of International Politics, New York, McGraw-Hill, 1979.
} 
Lo cierto es que, al trasplantar el problema de la anarquía internacional de una perspectiva antropocéntrica a una posición estructural, Waltz abre la puerta a la consideración de otros temas que conforman subsistemas, donde puede tener cabida el asunto de los derechos humanos. No obstante, en ninguno de los dos libros menciona el tema. Desde el ACD, el dato resulta de gran relevancia en cuanto a lo cuantitativo, pues demuestra que en la lógica del autor el asunto no forma parte de la producción intelectual respecto a la política internacional del momento. Esto es contrario al contexto histórico en el cual se estaba librando una batalla por encumbrar el entramado jurídico sobre el que descansan los principios mismos de la Organización de las Naciones Unidas.

Los textos de Waltz reflejan que su análisis se mantiene alejado de las preocupaciones que buscan dotar al sistema internacional de un sentido epistemológico, permeado por los derechos humanos. La macroposición es la que orienta sus aflicciones sobre el orden bipolar de la Guerra Fría, que se inscribe en la tradicional dinámica del poder. En su caso, los derechos humanos no son siquiera asumidos como una proposición de coherencia global.

En las postrimerías del siglo $\mathrm{xx}$, y sobre todo considerando el eminente final de la Guerra Fría, resulta obvio que las teorías de relaciones internacionales sufrieran cambios profundos en sus horizontes elementales. Así, surgieron una serie de postulados que buscaban explicar y predecir el comportamiento del sistema internacional ante los desafíos de un mundo fragmentado por la opinión pública, entre la unipolaridad y la multipolaridad.

Muchos de estos postulados son considerados dentro de la categoría de los textos clásicos debido a las referencias que han provocado y su relevancia en las discusiones al interior de la disciplina. Dentro de estas perspectivas innovadoras en el campo de las relaciones internacionales se encuentran las perspectivas feministas, siendo uno de sus máximos representantes el trabajo de Cynthia Enloe, Bananas, beaches and bases: making feminist sense of international politics. ${ }^{27}$ En éste, la autora cuestiona el rol preponderante con el cual se ha desarrollado la política internacional, en una clave altamente patriarcal.

$\mathrm{Su}$ análisis busca evidenciar las miradas que tejen a la disciplina con un sentido invisibilizado por la élite masculina. Sin dejar de admitir lo novedoso de su trabajo, para fines de nuestra investigación lo importante consiste en identificar los vínculos de su obra con los derechos humanos. La respuesta es exactamente la misma que la exhibida en Kenneth Waltz: Enloe no realiza

\footnotetext{
${ }^{27}$ Enloe, Cynthia, Bananas, Beaches and Bases: Making Feminist Sense of International Politics, Pandora Press, Harper-Collins, 1989.
} 
mención alguna de los derechos humanos en la totalidad de su exposición.

A diferencia de lo trabajado por Waltz, es posible intuir que las preocupaciones de Enloe se concentraron en evidenciar el dominio patriarcal en el que se desenvuelve la disciplina. Quizá por este motivo, los derechos humanos no tengan un rol en sus construcciones, ni como macroposición ni como proposición de coherencia global. Sin embargo, queda para el registro otro dato empírico que contribuye a la ratificación de nuestro argumento central.

Para finalizar este apartado, retomaremos dos textos: The Clash of Civilizations and the Remaking of World Order, ${ }^{28}$ de Samuel P. Huntington, y The End of History and the Last Man, de Francis Fukuyama. ${ }^{29}$ El primero de los dos surge a partir de un artículo publicado en Foreign Affairs, que condensa las ideas centrales de la obra. Huntington plantea que los conflictos venideros dejarán de librarse con base en la ideología, tal como sucedió durante la Guerra Fría. En el mundo contemporáneo, los conflictos se desarrollarían entre civilizaciones. Así, Huntington se encarga de gestar una serie de criterios que configuran determinadas probabilidades de que una civilización se enfrente con otra, haciendo una gran dicotomía entre la civilización occidental y el mundo islámico.

En realidad, el texto de Huntington generó una serie de críticas que se fueron condesando en un cuerpo sólido de opositores. No obstante, en su libro, Huntington dedica un subcapítulo al tema de los derechos humanos y menciona en 77 ocasiones la noción. Cualitativamente estas menciones se inscriben en un intento por explicar el universalismo occidental y la forma en que el discurso de la democracia y los derechos humanos son empleados precisamente para distinguirse de las otras civilizaciones.

En el texto de Huntington, se presenta una lógica que inscribe a los derechos humanos como una especie de carta de la baraja europea y estadounidense que buscan acomodar el juego de la política mundial a su favor. Los derechos humanos no son un asunto central para su concepción teórica, sino un elemento que diferencia a la cultura occidental de las otras culturas que describe Huntington. El ejemplo de este autor no conforma una macroposición, pero sí la proposición de coherencia global por excelencia.

Por otro lado, Fukuyama, quien sentó el precedente para las provocaciones de Huntington, de igual forma publicó sus postulados elementales en forma de artículo en el National Interest antes de desarrollar la mencionada obra. El autor plantea que, tras la finalización de la Guerra Fría, Occidente había

\footnotetext{
${ }^{28}$ Huntington, Samuel, The Clash of Civilizations and the Remaking of World Order, New York, Simon \& Shuster, 1996.

${ }^{29}$ Fuxuyama, Francis, The End of History and the Last Man, New York, Free Press, 1992.
} 
logrado posicionarse como el pináculo de la síntesis hegeliana y que, a partir de ese momento, no habría más disputas en el sentido en que discurrió la confrontación capitalismo-comunismo.

Bajo esta lógica, Fukuyama articula una propuesta nutrida de la filosofía que describe la expansión de los valores occidentales por el mundo. Es en este sentido que el autor rodea sus interacciones con los derechos humanos. En todo el texto, Fukuyama solamente habla en catorce ocasiones sobre derechos humanos, una cifra reducida considerando el contexto histórico en que se configura su argumento. Como ya hemos mencionado, el tema no es sustancial en sus postulados, solamente inscribe al discurso de los derechos humanos como un elemento de superioridad moral, por parte de Occidente, con respecto a sus enemigos en el mundo.

Esta visión triunfalista de las relaciones internacionales da por sentado que algunos valores conforman un instrumento de política exterior para los países que los poseen, entre los cuales se inscriben los derechos humanos. Esta es una proposición de coherencia global, pero de menor alcance que la presentada por Huntington.

Aunque la obra de Fukuyama ha perdido adeptos en el desarrollo del siglo XXI, sus postulados propusieron un influyente panorama para la teoría de relaciones internacionales de la pos Guerra Fría. No obstante, ni sus postulados ni los de Huntington hicieron el intento de proponer una ruptura ontológica o epistemológica sobre los derechos humanos como macroposición de las relaciones internacionales.

\section{Los libros de texto para internacionalistas y los derechos humanos}

La revisión detallada de los libros de texto seleccionados para la investigación demuestra que el tema de los derechos humanos en las relaciones internacionales es marginal para una gran parte de los trabajos. Sin embargo, se encuentran menciones en otros libros introductorios que matizarían esta idea de que el tema de derechos humanos es marginal en el mainstream académico internacionalista. En el primer bloque de libros revisados (13) queda de manifiesto que el tema no es central, incluso, en cuatro de ellos, llega a mencionarse el concepto sólo en una ocasión. (véase cuadro 2). 


\section{Cuadro 2}

Menciones a los derechos humanos en los libros de texto de las relaciones internacionales

\begin{tabular}{|l|c|}
\hline Libro & $\begin{array}{c}\text { Número de } \\
\text { menciones }\end{array}$ \\
\hline $\begin{array}{l}\text { 1. John A. Vasquez, The power of power politics. From Classical } \\
\text { Realism to Neotraditionalism, UK, Cambridge University Press 2004. }\end{array}$ & 1 \\
\hline $\begin{array}{l}\text { 2. Michael C. Williams, The Realist Tradition and the Limits of In- } \\
\text { ternational Relations, Cambridge, UK, Cambridge University Press, } \\
\text { 2005. }\end{array}$ & 1 \\
\hline $\begin{array}{l}\text { 3. Michael C. Williams (editor), Realism Reconsidered. The Legacy } \\
\text { of Hans Morgenthau in International Relations, New York, Oxford } \\
\text { University Press, 2007. }\end{array}$ & 1 \\
\hline $\begin{array}{l}\text { 4. Martin Griffiths, Realism idealism and international politics. A } \\
\text { Reinterpretation, London and New York, Routledge, 1992. }\end{array}$ & 1 \\
\hline $\begin{array}{l}\text { 5. Alan Cassels, Ideology and International Relations in the Modern } \\
\text { World, London and New York, Routledge, 1996. }\end{array}$ & 2 \\
\hline $\begin{array}{l}\text { 6. Robert, M. A., Crawford, Idealism and Realism International Re- } \\
\text { lations, Beyond the Discipline, London and New York, Routledge, } \\
\text { 2000. }\end{array}$ & 2 \\
\hline $\begin{array}{l}\text { 7. Thomas W. Smithh, History and International Relations, London } \\
\text { and New York, Routledge, 1999. }\end{array}$ & 3 \\
\hline $\begin{array}{l}\text { 8. Colin S. Gray, War, Peace and International Relations An Intro- } \\
\text { duction to Strategic History, London \& New York, Routledge, 2007. }\end{array}$ & 8 \\
\hline $\begin{array}{l}\text { 9. Jörg Friedrichs, European Approaches to International Rela- } \\
\text { tions Theory. A House with Many Mansions, London and New York, } \\
\text { Routledge, 2004. }\end{array}$ & 18 \\
\hline $\begin{array}{l}\text { 10. James Der Derian, Critical Practices of International Theory. Se- } \\
\text { lected Essays, London and New York, Routledge, 2009. }\end{array}$ & 18 \\
\hline $\begin{array}{l}\text { 11. N. J. Rengger, International Relations, Political Theory and the } \\
\text { Problem of Order, Beyond International Relations Theory?, London } \\
\text { and New York, Routledge, 2000. }\end{array}$ & 19 \\
\hline
\end{tabular}


12. Fred Chernoff Theory and metatheory in international relations, Concepts and Contending Accounts, New York, Palgrave Macmillan, 2007.

13. Charles R. Beitz, Political theory and international relations, New Jersey, Princeton University Press, 1979.

En los textos enlistados, se evidencia que sigue vigente la hegemonía discursiva que considera al poder, a la seguridad y a los intereses nacionales como los temas dominantes de las relaciones internacionales. Por lo tanto, no se puede hablar de un "cambio de valores" en el seno de la academia internacionalista del mundo anglosajón. Esto es relevante si se piensa que los libros de texto de relaciones internacionales están pensados para una amplia gama de estudiantes en las universidades de Estados Unidos.

Los estudios internacionales forman parte de lo que se denomina Liberal Arts e implican una serie de contenidos en los cursos que los alumnos van eligiendo conforme orientan sus estudios universitarios de acuerdo a sus intereses. Es decir, así como alumnos de historia pueden elegir cursos de economía política, habrá estudiantes de relaciones internacionales que elijan cursos de sociología de las religiones.

Con el fin de aportar una visión general a las teorías de las relaciones internacionales, los académicos anglosajones han reunido en diversos libros, de manera introductoria y sintética, los temas teóricos para conocimiento de alumnos de las ciencias sociales, en general, y de otras especialidades como las ingenierías o la filosofía de la ciencia. Esto contrasta con la retórica internacionalista de las naciones occidentales que han posicionado a los derechos humanos como vara conceptual para medir regímenes no democráticos ni liberales.

Si desde el mundo occidental se plantea la normalidad de la convivencia internacional a partir de valores cosmopolitas, y se ubica la defensa de los derechos humanos como condición fundamental para la transformación de la comunidad internacional, es de extrañarse que en el ámbito académico eso no se corrobore. Si los derechos humanos no forman parte de la centralidad discursiva de las relaciones internacionales como ciencia social significa que tardarán en conformarse como valor esencial de la política internacional.

Encontramos un segundo bloque de textos introductorios donde el tema de los derechos humanos es mucho más importante (véase cuadro 3). En los textos seleccionados aparecen menciones generales a los derechos humanos. Lo primero que resalta es que el concepto se ubica en la lista de "temas nuevos" de la política internacional y de las relaciones internacionales como discipli- 
na científica. Así, los derechos humanos estarían a la par de la migración, el cambio climático, los valores democráticos, la expansión del capitalismo y la incipiente emergencia de la sociedad civil global.

Si los derechos humanos son un tema nuevo para la disciplina, esto podría explicar su marginalidad en los libros de texto revisados. Sin embrago, en el canon internacionalista, se asume que los derechos humanos se consolidaron en las instituciones mundiales emanadas de la Segunda Guerra Mundial. Se aprecia aquí una clara contradicción: los derechos humanos son un tema nuevo para las relaciones internacionales, pero se les ubica como primordiales en la construcción del sistema internacional de la posguerra a partir de 1945.

Cuadro 3

Mayor número de menciones a los derechos humanos en los libros de texto de las relaciones internacionales

\begin{tabular}{|l|c|}
\hline Libro & $\begin{array}{c}\text { Número de } \\
\text { menciones }\end{array}$ \\
\hline $\begin{array}{l}\text { 1. Charles R. Beitz, Political theory and international relations, New } \\
\text { Jersey, Princeton University Press, 1979. }\end{array}$ & 25 \\
\hline $\begin{array}{l}\text { 2. Robert Jackson, Classical and Modern Thought on International } \\
\text { Relations, New York, Palgrave MacMillan, 2005. }\end{array}$ & 55 \\
\hline $\begin{array}{l}\text { 3. Peter Sutch and Juanita Elias, International Relations: The basics, } \\
\text { London \&t New York, Routledge, 2007. }\end{array}$ & 56 \\
\hline $\begin{array}{l}\text { 4. Martin Griffiths, International Relations Theory for 21st Century. } \\
\text { An Introduction, London \& New York, Routledge, 2007. }\end{array}$ & 58 \\
\hline $\begin{array}{l}\text { 5. Scott Burchill, Theories of international relations 3rd edition, } \\
\text { New York, Palgrave Macmillan, 2005. }\end{array}$ & 93 \\
\hline $\begin{array}{l}\text { 6. Griffiths, Martin, Terry O'Callaghan \& Steven C. Roach, Interna- } \\
\text { tional Relations Key Concepts, London \&t New York, 2002. }\end{array}$ & 137 \\
\hline $\begin{array}{l}\text { 7. Chris Brown with Kirsten Ainley, Understanding International } \\
\text { Relations 3rd edition, New York, Palgrave Macmillan, 2005. }\end{array}$ & 172 \\
\hline $\begin{array}{l}\text { 8. Andrew Linklater and Hidemi Suganami, The English School of } \\
\text { International Relations. A Contemporary Reassessment, Cambridge } \\
\text { UK, Cambridge University Press, 2006. }\end{array}$ & 210 \\
\hline $\begin{array}{l}\text { 9. David P. Forsythe, Human Rights in International Relations, } \\
\text { Cambridge UK, Cambridge University Press, 2006. }\end{array}$ & 2159 \\
\hline
\end{tabular}


Cabe aclarar que los libros aquí analizados se escribieron con el propósito de ser utilizados como textos introductorios para los estudiantes universitarios de las relaciones internacionales. Por lo tanto, la estrategia de dichos libros era describir el enfoque de cada teoría, precisar sus objetivos e intereses, explicar la aproximación metodológica, subrayar cuál es el énfasis ontológico y metodológico de cada teoría y mencionar a los autores relevantes de cada una. Destacan los cuatro últimos textos de la lista como los que mayores menciones tuvieron sobre la relación entre los derechos humanos y las relaciones internacionales. De los 42 libros, revisados sólo esos cuatro le otorgaron relevancia didáctica al tema.

El libro International Relations Key Concepts está pensado para ofrecer, a manera de glosario, los conceptos clave de las relaciones internacionales, así como para ilustrar algunos debates entre teorías. La pregunta con la que abre la introducción plantea "¿Por qué los Estados han decidido ahora delegar mucha de su autoridad al nivel internacional?" Y esta pregunta se responde, entre otras cosas, por los alcances de los derechos humanos en los últimos años.

Las menciones a los derechos humanos aparecen en muchos apartados, entre ellos "Coerción”, "Cosmopolitanismo", "Globalización” y en su apartado específico de la página 142 a la146. Ahí se indica con claridad que el concepto está asociado a la fundación de las Naciones Unidas. Más adelante, concluye que, a pesar de su difusión, la adhesión al respeto de los derechos humanos sigue siendo una voluntaria de los Estados nacionales. ${ }^{30}$

Understanding International Relations es también un libro didáctico y le dedica el capítulo 11 al tema de "Las Relaciones Internacionales y el individuo: derechos humanos, ley humanitaria y guerra humanitaria”. El problema que aborda el capítulo es el de los derechos humanos como la ampliación de los valores liberales a escala global, lo cual, a su vez, implicaría el goce humano de los derechos fundamentales. Al mismo tiempo, se discute el derecho de los Estados para intervenir por razones humanitarias cunado los derechos humanos de los pueblos o sociedades pudieran estar en riesgo.

En el libro The English School of International Relations, los derechos humanos aparecen frecuentemente como prerrequisito para el ideal de una sociedad internacional (international society). El concepto sociedad internacional se usa en esa corriente teórica para señalar que el sistema internacional, a través de tratados, acuerdos, comercio, valores, amplias y complejas

\footnotetext{
30 Griffiths, Martin, Roach, Steven y Solomon, Scotr, Fifty Key Thinkers in International Relations, New York - London, Routledge, 1999, pp. 142-146.
} 
relaciones, funciona más como una sociedad en construcción que como un sistema anárquico.

Finalmente, el único libro dedicado plenamente al tema, Human Rights in International Relations, se plantea como objetivo explicar "la evolución y estatus de los derechos humanos en las relaciones internacionales a principios del siglo xxı". Se sustenta en el ideal liberal de la buena sociedad, es decir, "aquella basada en el respeto por la equidad y la autonomía de los individuos”. Por lo tanto, es un libro que defiende una postura teórica determinada: el liberalismo.

Este último concluye citando a Michale Ignatieff, quien dijo que "desde 1945, nuestra imaginación moral ha sido transformada por el crecimiento de un lenguaje y de prácticas morales universales, expresadas especialmente en una cultura compartida de derechos humanos". El optimismo de la anterior cita no se corrobora con los demás libros analizados en este artículo, en los cuales el tema de los derechos humanos no indican un cambio epistemológico ni ontológico que nos permita hablar de un verdadero cambio cultural.

El ACD realizado tuvo la intención de identificar si esos los libros de texto con información general tomaban en consideración a los derechos humanos y si los vinculaban como problema para las teorías de las relaciones internacionales. De los nueve libros revisados se corrobora que hay un claro desequilibro entre aquellos que sí consideran importante el tema de los derechos humanos, quienes lo hacen medianamente y quienes profundizan más. No obstante, ninguno de los textos mantiene en su composición sustantiva a los derechos humanos, salvo los cuatro últimos del cuadro 3. Los resultados del ACD se enumeran a continuación:

- Son mínimos los ejemplos de teorías de las relaciones internacionales que interpretan los derechos humanos y la orientación ontológica y epistemológica de la teoría.

- Hay algunas menciones que problematizan la aspiración universalista de los derechos humanos.

- Algunos textos señalan el uso discursivo de los derechos humanos como estrategia legitimadora de la política exterior de las potencias occidentales.

- Algunos textos señalan el uso de los derechos humanos como justificación para las intervenciones humanitarias.

- Se mencionan en varios textos las violaciones de los derechos humanos como práctica de ciertos países (China, Corea del Norte, Iraq, Estados Unidos). 
En los textos analizados para este apartado, los derechos humanos ayudan a soportar macroposiciones como la globalización del modelo capitalista, el final de la Guerra Fría y las reorientaciones de la soberanía estatal. Sirven también de referencias a contados hechos históricos y, en algunos casos, como temas de ciertos autores emblemáticos en la disciplina.

Pero la macroposición más importante que se detectó en el ACD es la idea de soberanía y cómo ésta puede verse erosionada por la irrupción del discurso de los derechos humanos. Si la soberanía era una de las aspiraciones más importantes de cualquier Estado nacional, la agenda internacional por los derechos humanos podría poner en el banquillo de los acusados prácticamente a cualquier Estado nacional.

Por supuesto, se entiende que se trata de una novedad que quizá en el siglo xxI pueda fortalecerse. El hecho de que la protección de los derechos humanos se convierta en una lucha global y transnacional, en un escenario ideal, obligaría a reducir la discrecionalidad de las prácticas del poder en el nivel doméstico. Y esto hace que la vertiente realista sea la menos interesada por otorgarle relevancia a los derechos humanos.

Las vertientes teóricas que intentan ubicar a los derechos humanos como parte de la agenda internacional son, definitivamente, las liberales y las cosmopolitas. De aquí se desprende una pregunta: ¿Las teorías cosmopolitas de las relaciones internacionales (pos-Morgenthau) les otorgan realmente centralidad a los derechos humanos como eje de la política internacional? En estricto sentido, no. Lo que sí hacen las teorías cosmopolitas de las relaciones internacionales es buscar evidencia de los arreglos jurídicos y legales que demuestren la cooperación internacional para afrontar los retos globales. Éstos se entienden como los retos que inevitablemente incluyen a todos los Estados, tanto en sus consecuencias, como en la búsqueda de sus soluciones. Los autores cosmopolitas se refieren aquí al combate al hambre y la pobreza, al desarme nuclear, al problema del calentamiento global, a las regulaciones financieras y, en menor medida, a la expansión de los derechos humanos.

\section{Los libros sobre temas contemporáneos de relaciones internacionales y los derechos humanos}

En este último apartado, se abordan los libros de relaciones internacionales que versan sobre distintos temas de la disciplina y que fueron editados en el siglo XxI, desde diversas perspectivas teóricas. La selección comparte los criterios generales que ya han sido enunciados en el devenir de la investigación, 
con la exclusividad de que, respondiendo a la naturaleza de este apartado, se buscó que los temas selectos tuvieran un alto margen de diversidad. Por ello, ningún texto trata la misma temática. Se aplicó la metodología para el análisis de los textos, por lo cual se expondrán resultados tanto cualitativos como cuantitativos.

Así, formulados como libros que plantean temas de interés actual para las relaciones internacionales, su estudio detallado nos muestra claramente que los derechos humanos no son un tema interconectado de primer orden. Es decir, aunque existen obras que abordan propiamente la vinculación derechos humanos y relaciones internacionales, principalmente aquellos que trabajan con derecho internacional público, el tema es periférico.

Mientras los derechos humanos no conformen un entramado que sea considerado en cualquier área de estudio de la disciplina, no podemos hablar de que existe una ruptura epistemológica u ontológica con respecto al tema. En realidad, nuestra propuesta inicial de que aún no se observa un cambio de valores en las distintas manifestaciones de la disciplina, se ve reforzada con las aproximaciones contenidas en este apartado.

De esta manera, la primera cuestión que resalta en nuestro análisis es que ninguno de los textos revisados le dedica un capítulo entero al tema de los derechos humanos. Aunque este no es propiamente un estudio comparativo, es notorio que la consideración ofrecida al análisis de temas como el Estado, la soberanía, el poder, la guerra, entre otros por el estilo, ocupan un lugar preponderante en las obras.

Por otro lado, los derechos humanos y los temas de los libros listados no gozan de un vínculo particular. La relación que se hace, generalmente, va inscrita en una suerte de campo semántico, orquestado con la etiqueta de "temas globales”, lo cual configura una proposición de coherencia global. Tal como se expuso en el apartado anterior, los derechos humanos no inscriben una lógica profunda para el operar de las teorías de relaciones internacionales. Al menos no lo hacen desde perspectivas particulares.

En el siguiente cuadro, se presentan los diez libros revisados, especificando el tema que abordan y las menciones a los derechos humanos realizadas: 
Cuadro 4

Menciones a los derechos humanos en libros contemporáneos sobre relaciones internacionales

\begin{tabular}{|l|l|c|}
\hline Libro & Tema & $\begin{array}{c}\text { Total de } \\
\text { menciones }\end{array}$ \\
\hline $\begin{array}{l}\text { Mervyn Frost, Global Ethics. } \\
\text { Anarchy, Freedom and International } \\
\text { Relations, London \&t New York, } \\
\text { Routledge, 2009. }\end{array}$ & $\begin{array}{l}\text { Consideraciones éticas } \\
\text { en las relaciones } \\
\text { internacionales }\end{array}$ & 18 \\
\hline $\begin{array}{l}\text { James Der Derian, Critical practices } \\
\text { of International Theory, London \&t } \\
\text { New York, Routledge, 2009. }\end{array}$ & $\begin{array}{l}\text { Perspectivas críticas de la } \\
\text { política global }\end{array}$ & 18 \\
\hline $\begin{array}{l}\text { Colin Wight, Agents structures \&t } \\
\text { international relations. Politics as } \\
\text { Ontology, Cambridge, UK, Cambridge } \\
\text { University Press, 2006. }\end{array}$ & $\begin{array}{l}\text { Debate sobre las } \\
\text { aproximaciones } \\
\text { epistemológicas, } \\
\text { metodológicas y } \\
\text { ontológicas de las } \\
\text { relaciones internacionales }\end{array}$ & 2 \\
\hline $\begin{array}{l}\text { Thomas W. Smith, History and } \\
\begin{array}{l}\text { International Relations, London \&t } \\
\text { New York, Routledge, 2005. }\end{array}\end{array}$ & $\begin{array}{l}\text { Reposicionamiento de la } \\
\text { historia en la teoria de las } \\
\text { relaciones internacionales }\end{array}$ & 3 \\
\hline $\begin{array}{l}\text { Richard Ned Lebow, Coercion, } \\
\text { Cooperation and Ethics in } \\
\text { International Relations. London \&t } \\
\text { New York, Routledge, 2007. }\end{array}$ & $\begin{array}{l}\text { Argumentación sobre el } \\
\text { valor de la ética en política } \\
\text { exterior }\end{array}$ & 5 \\
\hline $\begin{array}{l}\text { Alan Cassels, Ideology and } \\
\text { International Relations, London \&t } \\
\text { New York, Routledge, 2003. }\end{array}$ & $\begin{array}{l}\text { Análisis del papel de la } \\
\text { ideología en la política } \\
\text { mundial }\end{array}$ & 2 \\
\hline $\begin{array}{l}\text { A. Nuri Yurdusev, International } \\
\text { Relations and the Philosophy of } \\
\text { History. A Civilizational Approach, } \\
\text { New York, Palgrave MacMillan, } \\
\text { 2003. }\end{array}$ & $\begin{array}{l}\text { Analiza el sistema } \\
\text { internacional a la luz del } \\
\text { concepto de civilización }\end{array}$ & 33 \\
\hline $\begin{array}{l}\text { Sarah Petcy, Mercenaries. The } \\
\text { History of a Norm in International } \\
\text { Relations. Oxford, Oxford University } \\
\text { Press, 2007. }\end{array}$ & $\begin{array}{l}\text { Explora el papel de los } \\
\text { mercenarios en la historia } \\
\text { y las normas que les } \\
\text { acompañan }\end{array}$ & 2 \\
\hline
\end{tabular}




\begin{tabular}{|l|l|c|}
\hline $\begin{array}{l}\text { Barry Buzan and Ole Wæver, } \\
\text { Regions and powers. The Structure } \\
\text { of International Security, Cambridge, } \\
\begin{array}{l}\text { UK, Cambridge University Press, } \\
2003 .\end{array}\end{array}$ & $\begin{array}{l}\text { Consideraciones sobre } \\
\text { la seguridad regional y } \\
\text { su desarrollo en la post } \\
\text { Guerra Fría }\end{array}$ & 11 \\
\hline $\begin{array}{l}\text { Pavlos Hatzopoulos and Fabio Petito } \\
\text { (editors), Religion in International } \\
\text { Relations The return from Exile, } \\
\begin{array}{l}\text { London \& New York, Palgrave } \\
\text { Macmillan, 2003. }\end{array}\end{array}$ & $\begin{array}{l}\text { Reposicionamiento de la } \\
\text { religión en las relaciones } \\
\text { internacionales }\end{array}$ & 18 \\
\hline
\end{tabular}

Como podemos observar, en la tabla anterior el número de menciones no excede las 33, por lo cual se sugiere que los derechos humanos, en relación a diversos temas de las relaciones internacionales, también conforman un asunto sumamente periférico.

Definitivamente, aunque existe un estrecho vínculo conceptual entre varios de los temas enunciados y los derechos humanos, la relación en sentido cuantitativo es bastante vaga. Ello desprende una revelación interesante, puesto que se refuerza el planteamiento de que los derechos humanos no están pensados como una macroposición, al menos no en una muestra significativa, en los temas cardinales con perspectivas teóricas de la agenda de las relaciones internacionales en pleno siglo xxI.

Por otra parte, el ACD refleja resultados muy similares a los obtenidos en el apartado anterior, por lo cual solamente enumeramos las consideraciones más relevantes:

- Los derechos humanos juegan un rol periférico en temas teóricos de las relaciones internacionales. Por tanto, se sostiene que no han logrado conformar una ruptura epistemológica ni ontológica en la disciplina.

- Los derechos humanos componen, un entramado que respalda la ética universalista manifiesta en la cultura occidental.

- En el siglo Xxi los derechos humanos se siguen abordando bajo la lógica dominante del capitalismo. Por tanto, están también altamente relacionados con las perspectivas desarrollistas y neodesarrollistas que rodean este orden epistemológico sistematizado jerárquicamente, así como desde las perspectivas globales que se pronunciaron en el mundo de la pos Guerra Fría. 


\section{Reflexiones finales}

La evidencia empírica expuesta en este artículo demuestra que los derechos humanos como tema central en los textos de las relaciones internacionales no han llegado a ser un proyecto intelectual y político consolidado. En este sentido, no se aprecia un cambio epistemológico ni ontológico en las teorías de las relaciones internacionales que equivalgan al gran cambio de valores que se esperaría en el mundo académico.

Para la mayoría de los internacionalistas y de los libros de texto de la disciplina, los derechos humanos siguen siendo tangenciales, a pesar de la importancia que supuestamente alcanzaron a principios del siglo xxI. La hegemonía del poder, del interés nacional, de la soberanía y del Estado como actor privilegiado de las relaciones internacionales no ha sido todavía debilitada como horizonte explicativo de la política internacional.

Lo máximo que se puede sugerir es que los derechos humanos tienden a erosionar la supremacía de dichos problemas. 0 , para decirlo de otra forma, los derechos humanos apenas han ayudado a re problematizar el poder, el interés nacional, la soberanía y el papel del Estado en la política internacional.

Lo cierto es que los textos elaborados en el siglo xxi han colocado a los derechos humanos como una proposición de coherencia global, a diferencia de los resultados obtenidos en la mayoría de los textos clásicos que se revisaron, los cuales ni siquiera los consideran en este estatus. Cabe preguntarse si la transición de la indiferencia respecto a los derechos humanos como proposición de coherencia global es indicio de una transformación que los constituya como una macroposición en la disciplina.

Responder con un enunciado afirmativo a esta proposición parecería una intuición lógica; sin embargo, hay que considerar una serie de factores contextuales que están dando nuevos horizontes para la disciplina. Generalmente, como ilustramos con Kuhn, los cambios paradigmáticos en la ciencia son efectuados a través de una orquestación que mucho tiene que ver con cambios profundamente políticos, y es precisamente lo que se observa hoy en día.

Como mencionamos en el primer apartado de esta investigación, los derechos humanos, por más que han sido considerados como un instrumento ideado por el capitalismo occidental para afianzar su sistema de valores a nivel mundial, tienen en su núcleo elementos subversivos que cuestionan las prácticas de sus mismos creadores. De ahí que en grandes extensiones de la vida práctica a nivel global se esté configurando una fuerza que abandera a los derechos humanos y los articula como un ente deseable. 
En los libros de relaciones internacionales este movimiento no pasa desapercibido. Aunque en la actualidad solamente se les considera tangenciales, el contexto que se vive tanto en las sociedades centrales para la producción académica como en las periféricas, puede generar un salto considerable que repunte la relevancia del tema en las relaciones internacionales.

\section{Bibliografía}

Acharya, Amitav y Buzan, Barry, Non-Western International Relations Theory. Perspectives on and beyond Asia, New York - London, Routledge, 2010.

CAmacho, Daniel, "Relaciones internacionales, movimientos sociales y derechos humanos", en Mercedes de Vega (coord.), Diplomacia cultural, educación y derechos humanos, México, Secretaría de Relaciones Exteriores, 2011.

Enloe, Cynthia, Bananas, Beaches and Bases: Making Feminist Sense of International Politics, Pandora Press, Harper-Collins, 1989.

Funuyama, Francis, The End of History and the Last Man, New York, Free Press, 1992.

Giacaglia, Mirta, "Hegemonía. Concepto clave para pensar la política”, Tópicos, núm. 10, 2002.

Gilpin, RoBert, War and Change in World Politics, Cambridge, Cambridge University Press, 1981.

Griffiths, Martin, Roach, Steven y Solomon, Scott, Fifty Key Thinkers in International Relations, New York - London, Routledge, 1999.

Hoffmann, Stantey, “An American Social Science: International Relations”, en Stanley Hoffmann (ed.), Janus and Minerva: Essays in the Theory and Practice of International Politics, Boulder, Vo., Westview Press, 1987, pp. 3-24.

Huntington, Samuel, The Clash of Civilizations and the Remaking of World Order, New York, Simon \& Shuster, 1996.

Kenedy, Paul, The Rise and Fall of the Great Powers, New York, Random House, 1987.

Keohane, Rober \& Nye, Joseph, Power and Interdependence, New York, Longman, 2012.

KoRnPRoBST, MARKUS, “International Relations as Rhetorical, Discipline: Toward (Re-) Newing Horizons", International Studies Review, núm. 11, 2009, pp. 87-108.

Kunn, Thomas, La estructura de las revoluciones cientificas, México, FCE, 2004.

ОсноА Bilbaо, LuIs, La carrera de Relaciones Internacionales en México: origenes y situación actual, Ciudad de México, Colmex - BUAP, 2011.

Morgenthau, Hans, Politics Among Nations, New York, Alfred A. Knopf, 1948.

Morgenthau, Hans, Human Rights and Foreign Policy, New York, Council on Religion and International Affairs, 1979. 
Waltz, Kenneth, Man, state and war. A theoretical analysis, New York, Columbia University Press, 1959.

Waltz, Kenneth, Theory of International Politics, New York, McGraw-Hill, 1979.

Weber, Cynthia, International Relations Theory: A critical Introduction, London \& New York, Routledge, 2005.

Wendt, Alexander, Social Theory of International Politics, Cambridge, Cambridge University Press, 1999.

ŽižEK, SLAVOJ, Ideología, un mapa de la cuestión, Buenos Aires, FCE, 2003. 